\title{
Hacia una visión integradora de la enseñanza y el aprendizaje de las matemáticas
}

\section{Towards an Integrative Vision of the Teaching and Learning of Mathematics}

\section{Abraham Arcavi ${ }^{1}$}

\begin{abstract}
Resumen. A partir de una definición informal de "perspectiva integradora" para la enseñanza de las matemáticas, este artículo propone diversos aspectos pasibles de integración y ejemplifica brevemente posibles vías de implementación en la escuela secundaria. La integración propuesta se refiere a aspectos usualmente disociados entre sí y en algunos casos hasta ignorados: diversos contenidos matemáticos (usualmente abordados como compartimentos estancos); conceptos y procedimientos; intuición y formalismo; matemáticas y la vida cotidiana. Concluimos reflexionando acerca de los posibles desafíos que presentaría la implementación de esta perspectiva.
\end{abstract}

Palabras clave: perspectiva integradora, conceptos, procedimientos, intuición, formalismo.

Fecha de recepción: 1 de febrero de 2018 . Fecha de aceptación: 3 de marzo de 2018

1 Departamento de Enseñanza de las Ciencias, Instituto Científico Weizmann (Israel) abraham.arcavi@ weizmann.ac.il 


\begin{abstract}
Following an informal definition of "integrative approach" for the teaching of mathematics, this paper proposes several aspects susceptible of integration and briefly exemplifies possible implementation ways in high school. The proposed integration refers to aspects which are usually dissociated and sometimes even ignored: mathematical content (usually addressed in a compartmentalized manner); concepts and procedures; intuition and formalism; mathematics and everyday life. We conclude by reflecting on the possible challenges in carrying on this approach.
\end{abstract}

Keywords: integrative approach, concepts, procedures, intuition, formalism.

\title{
INTRODUCCIÓN
}

Una de las metas generales del aprendizaje de las matemáticas es lograr que nuestros alumnos desarrollen un conocimiento flexible, ágil, competente y aplicable y que lo puedan revitalizar y regenerar continuamente adaptándolo a situaciones nuevas. Lamentablemente esta meta no es tan fácil de alcanzar y frecuentemente escuchamos lamentos de maestros, alumnos, padres y de la comunidad en general acerca de los fracasos de la educación matemática y sobre todo, acerca del rechazo que inspira esta disciplina, a la vez tan respetada y tan temida.

En este artículo, mi objetivo es proponer y analizar algunas prácticas de la enseñanza de matemáticas a las que considero consistentes con el logro de la meta descripta más arriba y que quizá, conjuntamente con otros cambios, puedan revertir, los aspectos y consecuencias menos felices de la educación matemática. Las prácticas que describiré por medio de ejemplos se centran en algunos de los múltiples aspectos de lo que definiríamos como una visión integradora de la educación matemática, aludiendo al mismo tiempo a lo que se sugiere integrar y cómo abordarlo.

Comencemos por repasar la definición de la palabra "integración". De acuerdo al Diccionario de la Lengua Española de María Moliner (segunda edición, 1999), podemos distinguir tres aspectos de este concepto:

- "hacer entrar una cosa en otra más amplia";

- "acción y efecto de hacer un todo o conjunto con partes diversas";

- "componer, constituir, formar". 
En lo que se refiere a integración de conocimientos, se puede decir que estos tres aspectos complementarios serían una versión breve de uno de los principios esenciales del constructivismo: aprender es coordinar, acomodar, asimilar un conocimiento por parte de un individuo (usando términos de Piaget, el constructivista por excelencia) al complejo sistema de sus conocimientos previos, combinando componentes para formar un nuevo todo funcional y dinámico. Es esta definición, y este enfoque brevemente expuesto (y quizás de manera simplista) lo que me ha guiado en la preparación de este artículo, que ofrezco como una oportunidad para la reflexión y el diálogo.

\section{INTEGRACIÓN DE CONTENIDOS}

"Si hoy es martes, esto es Bélgica" (If it's Tuesday, this must be Belgium, 1969) es el título de una comedia cinematográfica -una parodia a la industria del turismo organizado y sus consumidores-. El título alude al hecho de saber dónde se está solamente a través del día de la semana, tal cual lo indica el programa del paseo. En muchas ocasiones, este es un síndrome común en la enseñanza de las matemáticas: los alumnos "saben" cómo abordar un problema (y en general abordar un problema es casi sinónimo de escoger las fórmulas a aplicar), en base al capítulo del libro en el que aparece el problema. En estos casos, la contigüidad problema-fórmula suele ser una importante característica, si no el pilar, del proceso de enseñanza y aprendizaje de matemáticas. A veces, este fenómeno es tan marcado al punto de que es práctica común que en un examen que abarca varios tópicos, cada problema debe estar precedido por un título que especifique su tema, como una sugerencia implícita sobre los procedimientos a aplicar. Esta práctica difícilmente puede apoyar el desarrollo de un conocimiento flexible y ágil al que todos aspiramos, sin embargo, es mucho más frecuente de lo que nos imaginamos, y quizá, a veces, nosotros mismos la estimulemos inconscientemente.

Como parte de un proyecto para incentivar a alumnos con tendencias a desertar de las matemáticas, hemos coordinado con colegas un equipo de maestros para desarrollar un currículo con un enfoque pedagógico especial basado en las capacidades y necesidades de alumnos considerados con poca inclinación (y motivación) para las matemáticas. En el marco de este proyecto, ${ }^{2}$

\footnotetext{
2 Para una descripción de este proyecto, véase Arcavi, Hadas \& Dreyfus (1994) y Arcavi (2000).
} 
y con el objetivo de explorar la viabilidad de nuestros enfoques, hemos conducido numerosas clases experimentales, conversaciones con maestros, y entrevistas con alumnos. Uno de esos experimentos consistió en diseñar problemas que integren contenidos de distintas áreas y probarlos con alumnos. Por ejemplo, consideremos el siguiente problema:

El gráfico Cartesiano de las funciones $y=a x+1$ es una recta que forma un triángulo con los ejes coordenados. Lanzamos un dado común y sustituimos a por el valor obtenido $(1,2,3,4,5$, o 6$)$.

- ¿Para qué valor de a el triángulo formado es isósceles?

- ¿Para qué valor de a se obtiene el triángulo de menor área?

- ¿Cuál es la probabilidad de que el área del triángulo formado sea menor que 1/6?

- ¿Cuál es la probabilidad de que el área del triángulo formado sea mayor que 1/2?

Este problema requiere conocimientos básicos sobre funciones lineales, geometría y cálculo de probabilidades. Quizá la integración que pretendemos lograr mediante éste problema tenga la apariencia de una "mezcla" artificial de temas, pero consideramos que presenta al alumno el desafío de coordinar pensamientos, conectarse con los conceptos y considerar opciones en lugar de aplicar automáticamente una fórmula pre-establecida relacionada con el tema tratado. No describiré acá los intentos de solución que hemos recolectado en nuestras entrevistas con alumnos, cada uno de ellos con su manera idiosincrásica de pensar, sus aciertos y sus errores. Pero lo que sí quisiera compartir son los comentarios hechos por algunos alumnos cuando al concluir sus intentos de solución se les preguntó al respecto.

A1 se manifestó tensa y asustadiza al leer el enunciado y dijo "Yo no sé cómo resolver esto". La entrevistadora la tranquilizó y de a poco ella fue abordando el problema y logró resolverlo con algunos errores de cálculo y muchas dudas en el proceso. Al final, comentó su experiencia.

A1: "Las preguntas en clase son más fáciles. Nunca antes se me juntaron probabilidades con geometría analítica. Por ejemplo, en clase, hay una técnica para probabilidades, entonces yo sé que tengo que dibujar un "árbol". Acá es más abstracto, 
solamente cuando tracé [el bosquejo del gráfico] entendí lo que me pedían. ... Una pregunta como ésta con geometría no vi nunca, no tenía idea por dónde empezar. En clase yo sé que tengo que dibujar un árbol..."

Entrevistadora: "¿Acaso la pregunta te pide un árbol?"

A1: "No, pero así nos enseñaron, es el método de trabajo, acá no sabía cuál es el método."

A2: "Nunca antes estudié cómo enfocar esto, por eso me resultó difícil conectar las cosas."

Entrevistador: "¿Las preguntas son fáciles, difíciles?"

A2: "Me imagino que, si hubiera estudiado esto, lo hubiera podido resolver mejor" Entrevistador: "¿No estudiaste estos temas?"

A2: "Sí, pero no de esta forma"

Entrevistador: "¿Cuál es 'esta forma'?"

A2: "No sé, nunca antes estudié cómo ver ... las caras de un dado como una función"

Entrevistador: "¿Las preguntas son parecidas, distintas a las que ves en clase?"

A2: "Son distintas porque mezclan dos temas en una sola pregunta. En la clase, si es probabilidades, es eso sólo, si son funciones, es eso sólo.

Entrevistador: "iTe interesaría que haya preguntas como estas que conectan distintos temas?"

A2: "No, no creo. Me resulta más cómodo como es ahora. Es más simple. Pero, en realidad, si alguien me dice cómo hacer, yo diría, iay!, ¿cómo no lo entendí antes?"

A3: "Me resulta mejor estudiar como estudiamos en clase. Esto me parece raro... Lo dificil es que acá tengo que entender primero... Acá hay probabilidades y pendientes, áreas y geometría, no se centra en una sola cosa... Puede ser que si hubiera estudiado estas preguntas raras que tienen más o menos de todo me las hubiera arreglado bien..."

Notemos que los alumnos, si bien no muy exitosos en matemáticas, tienen un agudo poder de observación que se manifiesta en sus comentarios. Estos comentarios pueden ser muy instructivos para nosotros. Los alumnos no sólo notan las obvias diferencias entre este tipo de preguntas y las usuales de sus clases, sino que dentro de su limitada experiencia hacen una explícita caracterización de las mismas (combinación de temas, no hay una fórmula, primero hay que entender). Notemos que si bien les es más cómodo y fácil estudiar como lo han 
hecho hasta ahora, pueden enfrentar el problema, intentar resolverlo, y algunos lo logran sin errores. Pero lo más interesante es que los alumnos dejan entrever su sana intuición de que si hubieran estudiado de otra manera, hubieran tenido los elementos necesarios para abordar mejor estos problemas. El reclamo velado de estos alumnos es que ellos no pueden estudiar de una manera y ser examinados de otra. A este justo reclamo lo reinterpreto en términos de este artículo de la siguiente manera: no se puede pretender que la integración de conocimientos se produzca de manera espontánea por el mero hecho de confrontar a los alumnos con una situación que la requiere, esta integración debe ser parte indivisible de la práctica diaria del aula. Jugando un poco con las palabras y el metalenguaje, diría que la integración de conocimientos debe ser parte integral de la práctica pedagógica. A esa integración la debemos propulsar al menos en dos terrenos complementarios: en la selección de problemas apropiados (o si es necesario en el diseño de problemas nuevos) y en el tipo de preguntas y diálogos a sostener en las clases.

Hay muchas áreas de matemáticas que son candidatas a integración de conocimientos, ${ }^{3}$ textos que la promueven, docentes creativos que la estimulan. Nuestro desafío es ser conscientes de las posibilidades y del potencial educativo de su implementación, e intentar llevarlas a la práctica cotidianamente.

\section{CONCEPTOS Y PROCEDIMIENTOS}

"En el conocimiento matemático también se han distinguido dos tipos básicos: el conocimiento conceptual y el conocimiento procedimental. El primero está más cercano a la reflexión y se caracteriza por ser un conocimiento teórico, producido por la actividad cognoscitiva, muy rico en relaciones entre sus componentes y con otros conocimientos; tiene un carácter declarativo y se asocia con el saber qué y el saber por qué. Por su parte, el procedimental está más cercano a la acción y se relaciona con las técnicas y las estrategias para representar conceptos y para transformar dichas representaciones; con las habilidades y destrezas para elaborar, comparar y ejercitar algoritmos. El conocimiento procedimental ayuda a la construcción y refinamiento del conocimiento conceptual

\footnotetext{
${ }^{3}$ En Arcavi \& Hadas (2000) y en Arcavi (2008) presentamos problemas de geometría resueltos por medio del uso de funciones, en el cuales ejemplificamos como el conocimiento de cada uno de los dos temas se enriquece mediante la interconexión entre ellos.
} 
y permite el uso eficaz, flexible y en contexto de los conceptos, proposiciones, teorías y modelos matemáticos; por tanto, está asociado con el saber cómo."

"Estas dos facetas (práctica y formal) y estos dos tipos de conocimiento (conceptual y procedimental) señalan nuevos derroteros para aproximarse a una interpretación enriquecida de la expresión ser matemáticamente competente. Esta noción ampliada de competencia está relacionada con el saber qué, el saber qué hacery el saber cómo, cuándo y por qué hacerlo." (Vasco, 2005, p. 5)

Así expuesto, es sólo natural y lógico que ambos tipos de conocimientos sean esencialmente complementarios, no obstante, no parece serlo así para todos. Uno de los puntos álgidos de la discordia que se ha dado en llamar "las guerras de las matemáticas" que han tenido lugar en los Estados Unidos y en otros países, fue la toma de posiciones extrema a favor de la supremacía de uno de estos dos tipos de conocimientos. Aunque es claro que acá se impone la integración, una de las controversias centrales es, y sigue siendo, ¿̇a cuál de los conocimientos dar prioridad? ¿dónde poner el énfasis, en los conceptos o en los algoritmos?

Como parte de la respuesta que propongo, quiero presentar un ejemplo de un "producto" del razonamiento y procedimiento de un alumno que ilustra una sana integración entre estos dos tipos de conocimientos, a la que quisiéramos aspirar.

En el proceso de solución de la ecuación $\frac{2 x+3}{4 x+6}=2$, y en lugar de abalanzarse proceduralmente para producir una solución, un alumno se detuvo para hacer una 'lectura' de los símbolos. Notó que el numerador es siempre la mitad del denominador y, por ende, la fracción (en el lado izquierdo de la igualdad) nunca puede ser igual a 2, concluyendo que la ecuación carece de solución. No obstante haber resuelto el problema "conceptualmente", él quiso intentar resolver la ecuación, usando las conocidas reglas sintácticas, para ver cómo el procedimiento simbólico le confirmaría su hallazgo. Desafortunadamente, la manipulación técnica produce $x=-1 \frac{1}{2}$, en contradicción con lo obtenido anteriormente. El alumno quedó desconcertado por unos momentos, hasta que intentó sustituir ese valor en la ecuación original. Es entonces cuándo notó que el valor hallado, es precisamente el único que no es posible sustituir en la expresión simbólica (porque anula el denominador), y que esa es la manera en que el álgebra expresa la imposibilidad de solución. Tanto la inspección a-priori de los símbolos para 'sentir' el problema y su significado, como el a-posteriori intento de verificación y comparación de los significados con el resultado de las manipulaciones sintácticas, son ejemplos de lo que he llamado el sentido de los símbolos -symbol 
sense (Arcavi, 1994). El alumno subordinó sus conocimientos procedimentales a su razonamiento conceptual, y como resultado aprendió algo (o como se diría en inglés, tuvo un "insight") en ambos campos: conceptualmente, redescubrió en un contexto distinto la problemática de la división por cero. Proceduralmente, el alumno se hizo consciente de la "manera indirecta" en que el manejo simbólico le estaba indicando la inexistencia de solución. Pero, por sobre todo, el alumno vivenció la importancia de coordinar ambos conocimientos.

Este ejemplo ilustra que ser competente implicaría, entre otras cosas, el ejercicio de una transición bidireccional, oportunista y flexible entre el uso de acciones desprovistas de significado (como la aplicación automática de reglas y procedimientos) y la aplicación del sentido común y la búsqueda de significados. Dicho de otra manera, la competencia incluiría,

- la oportuna postergación de los significados a favor de una aplicación rápida y eficiente de un procedimiento, pero también, cuando sea necesario o cuando uno lo desee, proceder a

- la interrupción de una rutina automática con el objeto de cuestionar, reflexionar, conectar ideas, sacar conclusiones o elaborar nuevos significados -o como lo expresaría Freudenthal, "desatascar un automatismo" para desencubrir su origen, su significado y su propósito (Freudenthal, 1983, p. 469).

Transiciones significativas y flexibles dentro de un mismo problema entre acciones caracterizadas como pobres en significados y aquellas en que los significados juegan un papel central (y viceversa) es una competencia crucial, especialmente en álgebra. ¿Cómo desarrolla y aplica uno ese tipo de flexibilidad? Quizá sea éste el quid de la cuestión acerca de la contraposición entre procedimientos y conceptos. Sfard (2000), buscando explicar por qué las matemáticas son tan difíciles para tantos, describe la siguiente circularidad: si el significado (los conceptos, las ideas) es función del uso, uno debe manipular un concepto para entenderlo (en nuestro caso, manipular símbolos para "sentirlos" y sentir qué pueden hacer por nosotros), pero por el otro lado, ¿̇cómo podemos usar algo sin entenderlo (o sin sentirlo)? Sfard afirma que es precisamente esa circularidad lo que constituye una seria trampa para los alumnos, pero, ella sostiene que, al mismo tiempo esto es el combustible del proceso de aprendizaje. Sfard dice que, en este proceso, las formas y los significados, tal como son practicadas y vivenciadas por los alumnos, serían como dos piernas que hacen posible el caminar 
hacia adelante debido a que no están nunca en el mismo lugar, y en cada momento una de ellas está por delante de la otra.

Si adoptamos esta bonita metáfora, entonces es muy posible que el desarrollo de esta competencia, no sólo esté ligado a capacidades cognoscitivas, sino también a las actitudes que tengamos hacia el conocimiento y el aprendizaje. Lo que se desprende, por sobre todo, es que uno debe desarrollar la suficiente paciencia intelectual para convivir en armonía con la comprensión parcial y con la idea de que a veces los significados emergerán de lo que no tiene significado para nosotros (por ejemplo, como resultado de una práctica repetitiva, pero dentro de una cultura que apoya ocuparse de los significados). Otras veces, lo que carece de significado puede ser un subproducto o una condensación de una acción preñada de significados que aspiramos a automatizar para su efectivo uso futuro, aliviando así la carga a nuestra memoria.

Esta idea se corresponde con resultados de otros investigadores. Por ejemplo, Tobias (1990) investigó por qué reconocidos eruditos en el campo de las humanidades no eligieron el campo de las ciencias. Entre otras cosas, ella descubrió que para algunos de esos eruditos parte de la respuesta puede estar relacionada con la capacidad, o su ausencia, de convivir con la comprensión parcial por largos períodos de tiempo, hasta que los significados se conectan haciendo posible el surgimiento de una visión global. Parece que este es un ingrediente esencial del aprendizaje exitoso de las ciencias, mientras que las humanidades parecerían permitir un acceso más temprano a una visión global de contenidos y conceptos.

Esto nos lleva a proponer que el desarrollo de muchas de las competencias matemáticas deberá nutrirse de una cierta paciencia intelectual para convivir con comprensión parcial y una cierta confianza en que acciones futuras (no necesariamente sabiendo de antemano cuales y cuándo ocurrirán) avanzarán nuestra comprensión y nuestro conocimiento. Diría que esto implica tener una imagen muy distinta de aquella que considera a la comprensión como algo dicotómico (o la posees o no la posees) y al aprendizaje como una empresa rápida y sin esfuerzos para obtenerla. Convicciones como estas no suelen respetar el espacio y el tiempo necesarios para vivenciar y desarrollar plenamente el complejo y a veces lento desarrollo de las competencias matemáticas, y además desatiende el nivel de complejidad que estas competencias pueden tener.

Nuestras recomendaciones prácticas a este respecto consistirían en implementar "ambientes de aprendizaje enriquecidos por situaciones problema significativas y comprensivas, que posibiliten avanzar a niveles de competencia 
más y más complejos" (Vasco, 2005, p. 4) Estos ambientes deben dar lugar a consideraciones procedurales y conceptuales, a entablar diálogos reflexivos sobre problemas que aparentemente son sólo "técnicos", y a estimular a los alumnos a que verbalicen lo que piensan y sienten acerca de ciertos ejercicios (incluyendo sus intuiciones). ${ }^{4}$ Por sobre todo es importante tratar de evitar tanto los mensajes explícitos como los subyacentes que trasuntan impaciencia o necesidad perentoria de "cerrar" un tema, tratando, en cambio, de instilar simultáneamente la paciencia para convivir con entendimientos parciales y la confianza en que el trabajo continuo reforzará nuestra comprensión y nuestros logros.

En Friedlander \& Arcavi $(2012,2017)$ presentamos una colección amplia de tareas diseñadas con el propósito explícito de apoyar la integración de las destrezas técnicas con el conocimiento conceptual en el aprendizaje del álgebra.

\section{INTUICIÓN Y FORMALISMO}

Para muchos, las matemáticas consisten en abstracciones ininteligibles que están completamente divorciadas del sentido común. Quizá, sin darnos cuenta, nuestras pedagogías refuerzan esas convicciones ya que el aprendizaje no siempre hace uso efectivo e integral del sentido común, las intuiciones, y las formas perceptuales de conocer y contactarse con el mundo que todos tenemos (como por ejemplo la visualización). Generalmente, nos concentramos en los símbolos y sus leyes sintácticas, en generalizar propiedades y en expresarlas formalmente. En esta sección quiero presentar un ejemplo que no me canso de citar y repetir, y que me parece un hermoso modelo para dar un lugar de prominencia a la intuición de los alumnos, para trabajar con ella, respetarla, cultivarla al punto de que se transforme en un valioso recurso al servicio del desarrollo de nuestras competencias matemáticas.

Seymour Papert, el creador del lenguaje Logo de programación, presenta el siguiente problema (Papert, 1980, p. 144): Imaginemos una cinta colocada sobre el Ecuador del planeta Tierra. Para el propósito de nuestro problema consideremos al planeta como una esfera perfecta de aproximadamente 6,400 kilómetros de radio. Alguien nos propone alzar la cinta y colocarla sobre pilotes de 2 metros de alto. Esto obviamente implica que necesitaremos una cinta más larga. Pero, ¿cuánto más larga? Papert comenta que la mayoría de las personas tiene una

\footnotetext{
4 Véase, por ejemplo, el análisis de una conversación de clase descrito en Arcavi (2007).
} 
fuerte intuición de que se necesita agregar mucha más cinta. En realidad, el cálculo es muy simple, el exceso de cinta está dado por $2 \pi(R+h)-2 \pi R$ donde $R$ es el radio de la Tierra y $h$ la altura de los pilotes. El resultado es $2 \pi h$, es decir lo que se necesita agregar es sólo un poco más de 12 metros. Este resultado presenta dos sorpresas, la primera es que se necesita poca cinta y la segunda es que el resultado es independiente del radio de la Tierra, es decir, si hubiéramos comenzado con una naranja o con un planeta cien veces más grande que el nuestro, la cantidad de cinta adicional necesaria sería exactamente la misma. Estas sorpresas, sostiene Papert, deben ser cuidadosamente tratadas. Una reacción bastante común, por lo menos yo la he escuchado de algunos de mis maestros en condiciones similares, consiste en recalcar que nuestras intuiciones son imperfectas, que no debemos fiarnos de lo que nos parece, y que es sólo el instrumental matemático el que nos dará la respuesta correcta, aunque ésta nos parezca extraña. Sin duda, este es un pensamiento acertado, pero cuando se repite demasiado, es la receta segura para apresurar el divorcio entre nuestros alumnos y las matemáticas. Ellos comenzarán a sentir que sus ideas y las matemáticas son dos cosas totalmente ajenas, que las matemáticas no reflejan su sentido común, y por lo tanto no son "para mí". Papert sostiene que es nuestro deber pedagógico conectarnos con el sentido común de nuestros alumnos, hacerlo objeto de nuestra conversación, respetarlo y educarlo. ¿Cómo podríamos hacerlo en este caso? Papert nos propone basar su comunicación con la intuición (que la mayoría tiene sobre este problema) mediante el siguiente experimento de pensamiento visual.

Imaginemos que nuestro planeta es un cubo, su sección en el Ecuador sería entonces un cuadrado. Al elevar la cinta sobre pilotes de dos metros, la situación sería como la que muestra el siguiente esquema:

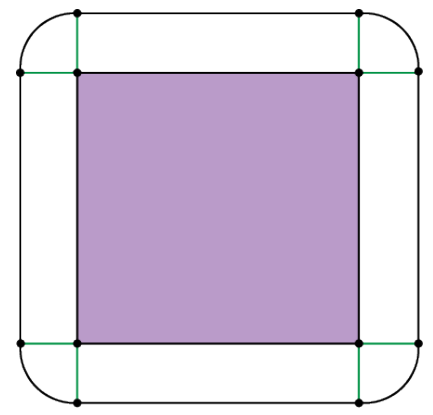


Se ve que en este caso la elevación de la cinta no requiere un largo adicional, excepto lo que se añadiría en las cuatro esquinas, que completan una circunferencia de radio 2 (altura de los pilotes) y que no depende del valor del lado del cuadrado. Si en lugar de un cuadrado, consideramos un octógono, la situación sería:

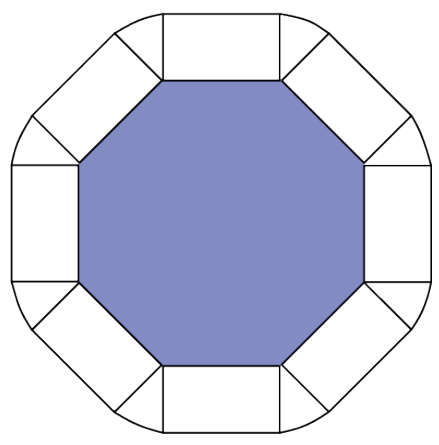

Similarmente, se ve que el largo adicional sigue siendo el equivalente a una circunferencia de radio 2, independientemente del largo del lado del octógono. Esto será lo mismo para un polígono de 20, 100, 1000 lados, y finalmente para un círculo.

La obligación pedagógica que se autoimpuso Papert fue descartar la opción que desdeña la intuición del alumno y, en cambio, buscar una forma de respetarla, dialogar con ella "en su mismo idioma", convencer sin usar una autoridad formal, precisamente para darle al formalismo un sustrato de sentido común.

Este ejemplo nos demuestra que es necesario y posible reconocer el conocimiento informal, respetar sus posibles desacuerdos con lo formal, integrarlo legitimizando su existencia convirtiéndolo en un participante activo del proceso de aprendizaje. En general, la visualización, en virtud de presentarnos imágenes tangibles y "concretas" puede ser un "factor esencial para crear la sensación de que algo es inmediato y evidente" (Fischbein, 1987, p. 101) y, por lo tanto, puede llegar a ser un poderoso aliado en nuestro intento de integrar lo informal y lo formal en el desarrollo de competencias matemáticas. ${ }^{5}$

\footnotetext{
${ }^{5}$ Para un análisis de varios ejemplos de visualización y sus posibles usos, véase Arcavi (2003).
} 


\section{LAS MATEMÁTICAS Y LA VIDA COTIDIANA}

Nuestras experiencias cotidianas nos confrontan con situaciones sociales, sicológicas, biológicas y hasta químicas y físicas. En cambio, excepto por el uso de la aritmética simple, porcentajes y proporcionalidad y quizá alguna consideración estocástica o estadística, nuestro mundo cotidiano aparenta ser matemáticamente pobre-ciertamente si se lo compara con la cantidad y la frecuencia de nuestras experiencias relacionadas con las otras disciplinas mencionadas.

Sin embargo, en nuestro mundo cotidiano hay tanta matemática como la que quisiéramos encontrar, si es que tenemos los medios y la predisposición de buscarlas. Es verdad, no están allí a ojos vista, llevamos la matemática con nosotros como utensilio para matematizar situaciones, no sólo por el placer de hacerlo, sino porque matematizar el mundo nos permite aprender sobre él, entenderlo más profundamente y moldear situaciones para mejorar nuestras vidas. Todo esto no necesariamente debe estar relacionado a situaciones de carácter científico, sino puede encontrarse en situaciones de la vida diaria de los alumnos.

Considérese, por ejemplo, la siguiente imagen de un columpio "transversal" fijado a una barra horizontal superior por medio de dos fuertes e inamovibles anillos.

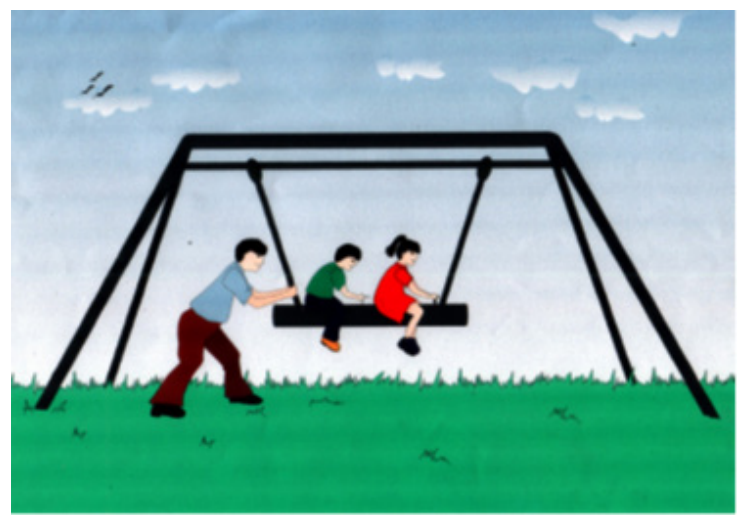

Un análisis geométrico (si lo miramos con esos ojos), nos permite determinar que este columpio es peligroso y que nunca debería construirse uno así. ¿Por qué? ¿Qué se debe corregir en este diseño para que el columpio sea seguro? 
Vayamos a un segundo ejemplo: una situación que recogí de la vida real. Imaginemos un curso (de cualquier asignatura) en el que las calificaciones de los alumnos en el examen final resultan muy por debajo de lo esperado, y por lo tanto el profesor decide aplicar un 'factor de corrección' para mejorar las calificaciones. Consideremos que las calificaciones son en la escala del 0-100 (100 correspondiendo a la calificación óptima), y el factor propuesto es $f(x)=10 \sqrt{x}$, donde $x$ representa la calificación original y $f(x)$ la corregida. Por ejemplo, si la calificación original fue de 64, la corregida será $10 \sqrt{64}$, es decir, 80 . Esta situación "real" abre una cantidad de preguntas importantes para los alumnos involucrados. Por ejemplo: Este factor de corrección ¿mejorará las notas de todos los alumnos? (para responder a esta pregunta es necesario comparar las funciones $f(x)=10 \sqrt{x}$ y $f(x)=x$, aunque algunos alumnos sugieren erróneamente que la respuesta es afirmativa porque la función de corrección es creciente). ¿Con este factor de corrección hay quiénes se beneficiarían más que otros? ¿Será este factor "socialmente justo"? ¿Elegirías tú un factor diferente? iProponlo y explica por qué lo prefieres! Esta situación-problema surgió fuera del contexto de los contenidos a estudiar en el aula, pero puede transformarse en un problema a tratar en clase. Este tipo de problema no sólo les interesaría a los alumnos por la importancia que tendría para ellos, sino que demuestra cómo instrumentos matemáticos pueden ayudar a comprender una situación, y hasta proveer argumentos acerca de lo justo o injusto de ésta. En resumen, sostengo que una mirada matemática al mundo que nos rodea nos revelará muchas más oportunidades matemáticas de las que nos imaginamos. Capitalizarlas e incorporarlas al proceso de enseñanza es otra de las integraciones que deberíamos intentar. $^{6}$

\section{CONSIDERACIONES FINALES}

En este artículo, ejemplifico algunas respuestas a las dos preguntas de su título, es decir, en una visión integradora ¿qué proponemos integrar? y ¿cómo? Hay muchos otros aspectos pasibles de integración, por ejemplo, la teoría y la investigación en educación matemática con la práctica de aula. Esto es un tema que merece no sólo un artículo aparte, sino quizá un libro entero. Y podríamos pensar

\footnotetext{
${ }^{6}$ Para un tratamiento más detallado de este aspecto, véase Arcavi (2002).
} 
en muchos otros. Pero me detendré aquí para hacer lugar a algunas consideraciones finales.

No quisiera dar la impresión que implementar una visión integradora es algo fácil. No bastan ejemplos de excelentes problemas, ni prácticas modelo de aula. Es un emprendimiento continuo, necesariamente dependiente del contexto socio-cultural local, y que requiere constante monitoreo. Además, podemos plantear preguntas de fácil formulación y cuya aparente simpleza puede encubrir serios dilemas. Por ejemplo: una visión integradora ¿es siempre deseable? Esta pregunta puede tener varios niveles y matices, quiero ejemplificar uno de ellos.

En una clase de un curso a nivel universitario sobre resolución de problemas en matemáticas que me tocó presenciar y analizar se distribuyó el siguiente problema: ¿qué día de la semana será el 26 de octubre de 2047? En este curso, el objetivo del profesor era analizar qué recursos matemáticos traían los alumnos para resolver este problema (por ejemplo, aritmética modular, sentido común, ensayo y error) y qué estrategias usarían para aplicar esos recursos. Es claro que la meta del problema, en este contexto, no es saber que se trata de un sábado, sino qué procesos y qué competencias matemáticas los alumnos emplean para saberlo. La pregunta quedó pendiente para la clase siguiente, en la cual una alumna trajo su respuesta correcta. Al preguntarle el profesor cómo la había hallado, la alumna respondió que lo hizo mediante una simple búsqueda con un programa computarizado de calendarios pasados y futuros. Sin duda un caso de feliz integración de competencias, de ingenio y de habilidad para encontrar de manera eficiente la respuesta de un problema. En un ambiente donde lo que se necesitaba era la respuesta, esta destreza hubiera sido ponderada. Sin embargo, en esta clase, la solución se produjo en flagrante violación de la meta del curso, y haciendo caso omiso a los "valores" culturales de esa clase que anteponen el proceso de resolución a la respuesta "a secas" del problema. Quizá la moraleja de esta pequeña historia sea que cuando tratamos de educación matemática, cualquier tema debe ser considerado de manera integral, es decir en relación al complejo sistema de valores y objetivos que enmarcan nuestras actividades. Además, diría que nuestra tarea de mejorar la educación matemática raramente nos eximirá de dilemas y preguntas, pero es esto, en mi opinión, parte del encanto de nuestra ocupación. 


\section{REFERENCIAS}

Arcavi, A. (1994). Symbol sense: informal sense-making in formal mathematics. For the Learning of Mathematics, 14(3), 24-35.

Arcavi, A. (2000). Problem driven research in mathematics education. Journal of Mathematical Behavior, 19(2), 141-173.

Arcavi, A. (2002). The Everyday and the academic in mathematics. In M. Brenner \& J. Moschkovich (Eds.), Everyday and Academic Mathematics in the Classroom. A Monograph edited by Journal for Research in Mathematics Education (pp. 12-29). Reston, Virginia: NCTM.

Arcavi, A. (2003). The role of visual representations in the teaching and learning of mathematics. Educational Studies in Mathematics, 52(3), 215-241.

Arcavi, A. (2007). El desarrollo y el uso del sentido de los símbolos. Uno, Revista de Didáctica de las Matemáticas, 44, 59-75.

Arcavi, A. (2008). Modelling with graphical representations. For the Learning of Mathematics 28(2), 2-10.

Arcavi, A. \& Hadas, N. (2000). Computer mediated learning: An example of an approach. International Journal of Computers for Mathematical Learning, 5(1) 25-45.

Arcavi, A., Hadas, N. \& Dreyfus, T. (1994). Engineering curriculum tasks on the basis of theoretical and empirical findings. In n J. P. Ponte $\&$ J. F. Matos (Eds.), Proceedings of the 18th International Conference on the Psychology of Mathematics (PME 18) (Vol. II, pp. 280-287). Lisbon: PME.

Fischbein, E. (1987). Intuition in Science and Mathematics: An Educational Approach. Dordrecht: Reidel.

Freudenthal, H. (1983) Didactical Phenomenology of Mathematical Structures. Dordrecht: Reidel.

Friedlander, A. \& Arcavi, A. (2012). How to Practice it: An Integrated Approach to Algebraic Skills. Mathematics Teacher 105(8), 608-614.

Friedlander, A. \& Arcavi, A. (2017). Tasks \& Competencies in the Teaching and Learning of Algebra. Reston, VA: National Council of Teachers of Mathematics (NCTM).

Papert, S. (1980) Mindstorms. New York: Basic Books.

Sfard, A. (2000) Symbolizing mathematical reality into being: How mathematical discourse and mathematical objects create each other. In P. Cobb, K. E. Yackel \& K. McClain (Eds.), Symbolizing and Communicating: Perspectives on Mathematical Discourse, Tools, and Instructional Design (pp. 37-98). Mahwah, NJ: Erlbaum.

Tobias, S. (1990). They're Not Dumb, They're Different. Stalking the Second Tier. Arizona: Research Corporation.

Vasco, C. (2005) Potenciar el pensamiento matemático: iUn reto escolar! - Estándares Básicos de Competencias en Matemáticas. Bogotá, D.C.: Ministerio de Educación Nacional. 